\title{
Mehr Empowerment!
}

Die Therapie bei Typ-1-Diabetes entwickelt sich rasant weiter, was eine Anpassung der Patientenschulung notwendig gemacht hat. Das neue Schulungs- und Behandlungsprogramm für ein selbstbestimmtes Leben mit Typ-1-Diabetes "PRIMAS" trägt dem Rechnung und wird derzeit im Alltag geprüft.

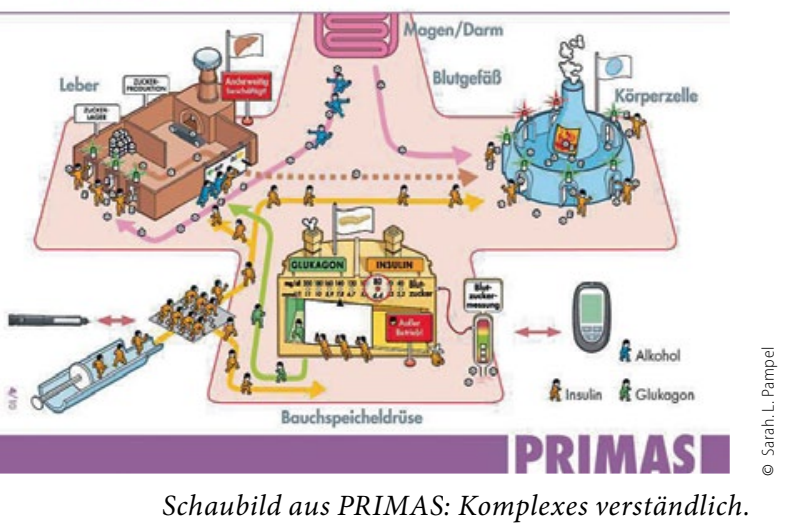

In den vergangenen 20 Jahren kamen neue Insuline und Therapiestrategien auf den Markt, die Behandlungsziele wurden neuen Erkenntnissen angepasst und das Empowerment der Patienten in den Mittelpunkt gerückt. Diese Entwicklung erfordert neue Schulungsinhalte und -formen. „Wissen allein hilft nicht, Patienten brauchen auch Erkenntnisse und Fähigkeiten“, so PD Dr. Bernhard Kulzer vom Forschungsinstitut der Diabetes Akademie Bad Mergentheim (FIDAM). Daher wurde seit 2007 auf Initiative des FIDAM und mit Unterstützung von Berlin-Chemie das neue Schulungsprogramm speziell für erwachsene Typ-1-Diabetiker PRI$\mathrm{MAS}^{*}$ entwickelt und ging im Sommer 2012 mit Train-the-Trainer-Seminaren bundesweit an den Start.

\section{"PRIMAS - ein Problemlöseprogramm"} Was ist neu bei PRIMAS, das sich als Erst- und als Wiederholungsschulung eignet? Es geht um individuelle Schulungsziele mit Fokus auf der Lösung individueller Probleme, Verbesserung der Lebensqualität, Motivation der Patienten zum engagierten Umgang mit dem Diabetes und um das Einbeziehen der Ange- hörigen. In Gruppen von 3-8 Personen lernen die Patienten in 12 Kursstunden á 90 Minuten über 6 oder 12 Wochen mithilfe moderner Materialien und tauschen sich aus. Dabei kann es um Basiswissen gehen, aber auch darum, wie Frau K. Blutzuckerspitzen und Herr S. Hypoglykämien in den Griff bekommt. „PRIMAS ist ein Selbstmanagementansatz, ein Problemlöseprogramm, es dient nicht nur der Wissensvermittlung“, so Kulzer.

Evaluiert worden ist PRIMAS in einer zur Publikation eingereichten randomisierten kontrollierten Studie gleichen $\mathrm{Na}$ mens, deren Ergebnisse bei der DDGJahrestagung 2012 vorgestellt wurden. In ihr wurde das neue Programm bei 161 Patienten aus 28 Praxen erfolgreich mit einer etablierten ICT-Schulung verglichen. Bei PRIMAS-Patienten sank der $\mathrm{HbA}_{1 \mathrm{c}}$-Wert im Gegensatz zur Kontrollgruppe, in der er stagnierte, schwere Hypoglykämien waren seltener und die Therapiezufriedenheit nahm zu. Die Ergebnisse dienen als Grundlage für die Zertifizierung durch die DDG sowie durch das Bundesversicherungsamt zur Aufnahme in das DMP Diabetes Typ 1, wie Kulzer berichtet hat. Mit der Akkreditierung und einer Abrechnungsziffer ist im Lauf des Jahres $2013 \mathrm{zu}$ rechnen.

Praxisstudie prüft Alltagstauglichkeit Zusätzlich wurde eine Praxisstudie gestartet, die das Programm über sechs Monate in 35 Schwerpunktpraxen im Alltagseinsatz prüft. Die Primas-in-Praxi-Studie mit 200 Patienten soll klären, ob sich die positiven Ergebnisse der kontrollierten Studie in den Alltag übertragen lassen. Die Ergebnissen werden laut Kulzer Mitte 2013 erwartet.

Das Schulungsmaterialen von PRIMAS bestehen u.a. aus Patientenbuch, Arbeitsblättern und Tabellen für Patienten sowie einem Schulungskoffer mit CD-ROM oder Folienordner und einem Curriculum für das Schulungspersonal.

*abgeleitet aus „Schulungs- und Behandlungsprogramm für ein selbstbestimmtes Leben mit Typ-1Diabetes". Die Analogie zum lateinischen 'primus' weckt die Assoziation zum Typ-1-Diabetes. Infos: www.primas-schulungsprogramm.de

Sarah Louise Pampel, München

Quelle: Satellitensymposium DDG-Herbsttagung, 17.11.2012, Berlin, Veranstalter: Berlin-Chemie

\section{SGLT-2-Hemmer allein oder kombiert}

\section{Oberste Ziele einer individuell angepassten Diabetestherapie sind das Vermeiden von Hypoglykämien und Gewichtszunahme. Mit dem ersten Vertreter einer neuen Wirkstoffklasse gelingt das gut.}

Die stringente und sichere $\mathrm{HbA}_{1 \mathrm{c}}$-Einstellung sollte früh nach der Diabetesdiagnose erfolgen, so Prof. Wolfgang Schmidt, Bochum. Bei Typ-2-Diabetes stehen dafür außer Metformin verschiedene Anti- diabetika zur Verfügung. Mit Dapagliflozin (Forxiga ${ }^{\circledR}$ gibt es nun den ersten zugelassenen Vertreter der insulinunabhängig wirkenden SGLT-2-Hemmer. Der selektive, reversible Inhibitor des Gluko- serücktransports in der Niere entfernt überschüssige Glukose insulinunabhängig über den Urin und ist als Monotherapie bei Metformin-Unverträglichkeit oder kombiniert mit anderen Antidiabetika inklusive Insulin zugelassen. In klinischen Studien senkte das Gliflozin Blutzucker, Gewicht und Blutdruck. Als unerwünschter Nebeneffekt traten vermehrte Candida-Genitalinfekte auf.

Ulrike Tietze, Berlin

Quelle: Satellitensymposium bei der DDGHerbsttagung 17.11.2012 in Berlin, Veranstalter: BristolMyers Squibb und AstraZeneca 